\title{
PROGNOSTIC CLINICAL, MORPHOLOGICAL AND IMMUNOHISTOCHEMICAL MARKERS OF LOW-GRADE SEROUS OVARIAN CARCINOMAS OF WOMEN WITH SEROUS BORDERLINE TUMORS IN ANAMNESIS
}

\author{
Varvara Hryhorenko \\ Postgraduate Student at the Department of Pathological Anatomy, \\ Kharkiv Medical Academy of Postgraduate Education, Ukraine \\ e-mail: drvaria94@gmail.com,orcid.org/0000-0001-6923-5906
}

\section{Summary}

The problem of finding reliable clinical and morphological criteria for diagnosis and prognosis of serous borderline ovarian tumors, their relationship with the risk of developing of low-grade serous ovarian carcinomas (LGSC) in future is still relevant.

This study is devoted to research and highlight of precursors that allow dividing women with serous borderline ovarian tumors (SBOT) into risk group for occurrence of LGSC within next 5 years.

The study included 22 patients with FIGO stage I-II SBOT aged 24 to 46 years, $9(39.13 \%)$ of whom were diagnosed with high-grade serous ovarian carcinoma next 5 years. Two study groups were formed: the control group $(n=13)$, which included patients with SBOT without further development of LGSC, and the main group $(n=9)$, which included women with emerging LGSCs. We studied expression of immunohistochemical markers Ki-67, MMP-9, p53, Bcl-2, E-cadherin, and also a diameter and localization of the tumor.

As a result of the study, it was found that patients with diameter of the SBOT $\geq 10 \mathrm{~cm}$ $\left(\chi^{2}=6.0, p<0.03\right)$, FIGO stage II $\left(\chi^{2}=4.7, p<0.03\right)$ and Ki-67 expression $\geq 10 \%\left(\chi^{2}=9.03\right.$, $\mathrm{p}<0.03$ ) have a high risk of developing LGSC within next 5 years. In the group of women who underwent LGSC development, there was a tendency to more pronounced expression of MMP-9 $\left(\chi^{2}=4.18, \mathrm{p}<0.04\right)$ and moderate and pronounced expression of Bcl-2 $\left(\chi^{2}=9.66\right.$, $\mathrm{p}=0.008)$.

According to our data, markers Ki-67, MMP-9, Bcl-2 are prognostic and can be used as markers of LGSC risk in women with a history of SBOT. Tumor diameter $\geq 10 \mathrm{~cm}$ is also a predictor.

Keywords: SBOT, prognostic markers, LGSC, Ki-67, MMP-9, Bcl-2, p53

DOI: https://doi.org/10.23856/4821

\section{Introduction}

Borderline ovarian tumors (BOT) were first described by Howard Taylor in 1929 and had characteristics of "semi-malignant", since despite tendency to disseminate along peritoneum, they had a favorable prognosis. In 1971, the International Federation of Obstetrics and Gynecology (FIGO) also implemented this group of neoplasms in classification, calling them "tumors with low malignancy potential" (Hauptmann et al., 2017).

BOT stands at $15-20 \%$ of all tumors of the mentioned localization, while in histological structure the leading place (from 53 to $65 \%$ ) is given to serous borderline ovarian tumors. (Davydova et al., 2021). Approximately 30\% of SBOT are bilateral (Davydova et al., 2021) 
(Ozer et al., 2012) Women of reproductive age in the age range of 30-40 years are susceptible to development of SBOT, (Davydova et al., 2021), the average age of patients, according to $\mathrm{WHO}$, is 43 years old (Herrington, $C$. S., 2020). In contrast to malignant neoplasms of the ovaries, $75 \%$ of SBOT is diagnosed at an early stage (IA, IB stages according to FIGO) (Hauptmann et al., 2017).

According to $2020 \mathrm{WHO}$ classification of tumors of female reproductive system, a small percentage of women with low-grade serous carcinomas had a history of SBOT. Although, according to WHO data, LGSC stands at only $5 \%$ of all ovarian carcinomas, an interesting and clinically important aspect is the identification of possible objective clinical and morphological predictors of LGSC occurrence in patients with a history of SBOT.

Despite the relatively favorable prognosis of PCOS, there is still no consensus on the criteria for aggressiveness and prognostic markers for these neoplasms. The disputability of this section of tumors of the female reproductive system is also confirmed by repeated changes in the classification of the WHO.

Numerous previous studies have shown that tumor size, FIGO stage, morphometric parameters (nuclear area, cell area, nuclear-cytoplasmic ratio) and expression of certain immunohistochemical markers can serve as prognostic criteria for malignant and borderline tumors.

The aim of our study was to detect prognostic criteria for LGSC occurrence in patients with PCOS anamnesis I and II according to FIGO.

\section{Materials and methods}

\subsection{Clinical material}

Clinical material in our study was medical cards, paraffin blocks and slides of patients who underwent bilateral or unilateral oophorectomy in the period from 2013 to 2018 at the Kharkov Regional Clinical Oncological Dispensary, Kharkov Institute of Medical Radiology and Oncology named by S.P. Grigoriev and Kharkov Military Medical Clinical Center of Northern Region. The study included 22 tumors: 13 SBOT without subsequent development of LGSC within 5 years (control group) and 9 SBOT of patients in whom LGSC was found in the next 5 years (study group).

The histological type of tumors was determined on the basis of viewing slides stained with hematoxylin-eosin according to the standard technique.

SBOT and LGSC were staged according to FIGO classification. The age of patients and the diameter of the tumor were taken from pathologists conclusion. The division of tumors by diameter into samples $<10 \mathrm{~cm}$ and $\geq 10 \mathrm{~cm}$ was performed in accordance to the study by Hatice Ozer et al. (Ozer et al., 2012). From each clinical case, 1 block was selected for immunohistochemical (IHC) studies.

\subsection{Immunohistochemical studies}

The material for research by immunohistochemistry method was fixed with $10 \%$ neutral formalin for $24 \mathrm{~h}$, embedded in paraffin, $4 \mu \mathrm{m}$ sections were prepared, which were applied to highly adhesive Super Frost glasses and dried at $37^{\circ} \mathrm{C}$ for 18 hours. De-masking heat treatment was performed by boiling the sections in citrate buffer ( $\mathrm{pH} \mathrm{6.0).} \mathrm{To} \mathrm{visualize} \mathrm{primary} \mathrm{antibodies,}$ an UltraVision Quanto Detection Systems HRP Polymer (Thermo scientific) detection system was used. DAB (diaminobenzidine) was used as a chromogen. 
Primary monoclonal antibodies (MCAT) from DAKO (Denmark), TermoScientific (Germany) and Diagnostic BioSystems (USA) were used. The expression of such markers was studied: Ki-67 (MiB-1, Termo Scientific), MMP-9 (Collagenaza IV, Diagnostic BioSystems), p53 (SP5, Termo Scientific), Bcl-2 (clone124, Dako Cytomation), E-cadherin ( EP7004, Termo Scientific).

To assess intensity of immunohistochemical label of markers Bcl-2, E-cadherin and MMP9, a semi-quantitative scale $0-3+$ was used: 0 - no expression, +- weak, ++- moderate, +++- pronounced reaction. To assess the immunohistochemical label of $\mathrm{p} 53$, percentage of nuclei staining was considered: $0=$ no staining, $1=<10 \%$ of nuclei, $2=10-50 \%, 3=51-80 \%$ and more than $80 \%=4$.

To assess the immunohistochemical label $\mathrm{Ki}-67$, percentage of stained nuclei was considered: $\leq 10 \%$ - absence or very weak proliferative activity, $11-50 \%$ - weak proliferative activity, $51-80 \%$ - moderate proliferative activity, more than $80 \%$ - high proliferative activity.

\subsection{Statistical analysis}

The relationship between the studied traits was researched using the nonparametric Pearson test with Yates' correction. The nonparametric Mann-Whitney test was used to process data of immunohistochemical studies and study relationship between the signs. A significance level of $\mathrm{p} \leq 0.05$ was considered significant.

\section{Results and discussion}

The patients' age in the control group varied from 24 to 44 years, the median was $39.54 \pm 1.47$ years. The age range study group was from 32 to 46 years, the average age in this group is $39.33 \pm 1.27$. Age differences in two groups were insignificant ( $p>0.05)$. The age of occurrence of SBOT in the study groups was significantly lower than in Cirstea`s A.E. et al. study, where the average age was 47.1 years (Cirstea et al., 2018), and according to the World Health Organization data (Herrington, 2020), which can be explained by the characteristics of a particular sample.

Table 1 presents the main clinical and morphological features of the patients.

Table 1

Clinical and pathohistological characteristics of the studied cases

\begin{tabular}{|c|c|c|c|c|}
\hline & \multicolumn{2}{|c|}{ Serous borderline ovarian tumors } & \multirow[b]{2}{*}{ Significance } \\
\hline & & $\begin{array}{c}\text { Control group } \\
(\mathrm{n}=13)\end{array}$ & $\begin{array}{c}\text { Study group } \\
(\mathrm{n}=9)\end{array}$ & \\
\hline Age & & $39,54 \pm 1,47$ & $39,33 \pm 1,27$ & $p>0,05$ \\
\hline \multirow{3}{*}{ Localization } & Right & $6(46,1 \%)$ & $4(44,4 \%)$ & \multirow{3}{*}{$\chi^{2}=4,3, p=0,1$} \\
\hline & Left & $3(23,1 \%)$ & $5(55,6 \%)$ & \\
\hline & Bilateral & $4(30,7 \%)$ & - & \\
\hline \multirow{2}{*}{ Size } & $<10 \mathrm{~cm}$ & $11(84,6 \%)$ & $3(33,3 \%)$ & \multirow{2}{*}{$\chi^{2}=6,0, p<0,03$} \\
\hline & $\geq 10 \mathrm{~cm}$ & $2(15,4 \%)$ & $6(66,7 \%)$ & \\
\hline \multirow{4}{*}{ FIGO stage } & I & $9(69,2 \%)$ & $2(22,2 \%)$ & \multirow{4}{*}{$\chi^{2}=4,7, p<0,03$} \\
\hline & II & $4(30,8 \%)$ & $7(77,8 \%)$ & \\
\hline & III & - & - & \\
\hline & IV & - & - & \\
\hline
\end{tabular}


According to the literature (Herrington, 2020), approximately one third of SBOT is bilateral. In our study, the proportion of bilateral tumors in the control group accounted for $30.7 \%$ of cases, which roughly coincides with the indicated information. We did not find statistically significant differences between tumor localization and the probability of LGSC occurrence in the future $\left(\chi^{2}=4.3, \mathrm{p}=0.1\right)$.

Tumor diameter less than $10 \mathrm{~cm}$ was observed in $11(84.6 \%)$ cases in the control group and in $3(33.3 \%)$ cases in the main group. A tumor of $10 \mathrm{~cm}$ or more in diameter was found in $2(15.4 \%)$ cases in the control group and in $6(66.7 \%)$ cases in the main group. When comparing the two groups, it was revealed that SBOT with subsequent development of LGSC is associated with a size of $\geq 10 \mathrm{~cm}(\mathrm{p}<0.03)$. Our research findings are consistent with those of Ozer H. et al. (Ozer et al., 2012) There is also a direct strong correlation between the size of the tumor and its proliferative activity $\left(\mathrm{r}_{\mathrm{s}}=0.84, \mathrm{p}<0.01\right)$.

The development of LGSC in women with a history of SBOT is associated with FIGO stage II $(\mathrm{p}<0.03)$.

Table 2 shows distribution of cases according to the degree of expression of the immunohistochemical label Ki-67 and p53.

Table 2

Distribution of cases by the degree of expression of the immunohistochemical label Ki-67 and p53

\begin{tabular}{|c|c|c|c|}
\hline IHC-marker & $\begin{array}{c}\text { Control group } \\
(\mathbf{n = 1 3})\end{array}$ & $\begin{array}{c}\text { Study group } \\
(\mathbf{n = 9})\end{array}$ & Significance \\
\hline & $\mathbf{K i - 6 7}$ & & $\chi^{2}=9,03, p<0,03$ \\
\hline 0 & 5 & 0 & \\
\hline$<10 \%$ & 4 & 3 & \\
\hline $10-50 \%$ & 3 & 5 & \\
\hline $51-80 \%$ & 1 & 0 & \\
$\geq 80 \%$ & 0 & & $\chi^{2}=2,96, p=0,2$ \\
\hline & $\mathbf{P 5 3}$ & 6 & \\
\hline 0 & 5 & 3 & \\
\hline$<10 \%$ & 5 & 0 & \\
\hline $10-50 \%$ & 3 & 0 & \\
\hline $51-80 \%$ & 0 & 0 & \\
\hline $81 \%$ & 0 & & \\
\hline & & & \\
\hline
\end{tabular}

Ki-67 is a protein, whose expression is traditionally associated with the proliferative activity of tumors (Menon et al., 2019) and their aggressiveness (Li et al., 2015) (Sun et al., 2018). In our study, the SBOT group with subsequent development of LGSC showed a significantly higher expression of Ki-67 compared to the control group with an increase of number of observations with marker expression more than $80 \%(n=5 / 9)$. When comparing the study group with the control group, it was revealed that SBOT, which were subsequently associated with the development of LGSC, is characterized by $\mathrm{Ki}-67$ expression $\geq 10 \%$ $\left(\chi^{2}=7.24, \mathrm{p}<0.007\right)$. So, the expression of $\mathrm{Ki}-67<10 \%$ was observed among the control group in $9(69.2 \%)$ cases out of 13 , and in the study group it was in $11.1 \%(n=1 / 9)$. According to many studies, level of $\mathrm{Ki}-67$ expression is a more sensitive predictor of malignant phenotype 
and possible tumor recurrence compared to its size. (Lowe et al., 2012) (Grimm et al., 2019) (Gottwald et al., 2013). Also, according to the study by Yu N. et al., Increased expression of $\mathrm{Ki}-67$ and E-cadherin play an important role in oncogenesis and progression of serous borderline tumors (Yu et al., 2017).

Numerous studies have noted the important role of p53 gene as a tumor suppressor due to the control of cell proliferation (Jin, 2005). Mutations in this gene are most likely genetic breakdown, found in many human tumors; therefore, level of p53 expression is one of the most studied in immunohistochemical panel for malignant neoplasms diagnosis of various localization (Corney et al., 2008). A few studies indicate a high frequency of p53 mutations in poorly differentiated serous ovarian carcinomas and a rare occurrence of such genetic abnormalities in LGSC and SBOT. (Zeren et al., 2014) (Corney et al., 2008). Many researchers describe more pronounced expression of p53 in SBOT and serous ovarian carcinomas in its absence in benign and borderline serous tumors of female gonads (Kaygusuz, 2011) (Ozer et al., 2012). In our study, there was a weak and moderate degree of p53 expression in SBOT without further development of LGSC, while in SBOT group with the subsequent development of highly differentiated serous ovarian carcinoma, expression p53 was absent in $6(66.67 \%)$ cases or there was a weak degree of expression in $3(33.33 \%)$ cases. Changes in p53 expression were not significantly different in two study groups $(\mathrm{p}=0.2)$.

Table 3 shows the expression intensity of markers MMP-9, bcl-2 and E-cadherin.

Table 3

Distribution of cases by the intensity of expression of IHC markers MMP-9, bcl-2, and E-cadherin

\begin{tabular}{|c|c|c|c|}
\hline IHC-marker & $\begin{array}{c}\text { Control group } \\
(\mathbf{n = 1 3})\end{array}$ & $\begin{array}{c}\text { Study group } \\
(\mathbf{n = 9})\end{array}$ & Significance \\
\hline & MMP9 & 3 & $\chi^{2}=4,18, p<0,04$ \\
\hline 0 & 10 & 6 & \\
\hline Low & 3 & 0 & \\
\hline Moderate & 0 & 0 & \\
\hline Pronounced & 0 & & $\chi^{2}=9,66, p=0,008$ \\
\hline & Bcl-2 & 0 & \\
\hline 0 & 0 & 0 & \\
\hline Low & 6 & 2 & $\chi^{2}=9,89, p=0,007$ \\
\hline Moderate & 5 & 7 & \\
\hline Pronounced & 2 & 0 & \\
\hline & E-cadherin & 4 & \\
\hline 0 & 0 & 5 & \\
\hline Low & 0 & 0 & \\
\hline Moderate & 5 & & \\
\hline Pronounced & 6 & & \\
\hline & & & \\
\hline
\end{tabular}

Matrix metalloproteinase MMP-9 is a remodeling endopeptidase that promotes local invasion and metastasis in tumors (Pârvănescu et al., 2015). It is one of the main biomarkers of serous ovarian cancer. (Huang H., 2018) According to literature, in serous borderline ovarian tumors, lower expression of MMP-9 is observed than in serous cancers (Sakata, 2000). 
In our study, the PCOS group with the subsequent development of highly differentiated serous carcinomas was characterized by a more pronounced expression of MMP-9 $(p<0.04)$. Weak expression was observed in the main group in $6(66.67 \%)$ cases out of 9 , while the control group was characterized by the absence of MMP-9 expression in 10 (76.92\%) cases out of 13 . The obtained results, in our opinion, may indicate malignancy of the SBOT followed by the development of LGSC with an increase in the level of MMP-9 expression. Such fact requires more close clinical observation of the patient.

Graph 1 shows a direct strong correlation between the expression level of Ki-67 and MMP-9 of the studied tumors $\left(r_{s}=0.84, p<0.05\right)$. This dependence, in our opinion, may be associated with a higher capacity of SBOT for microinvasion.

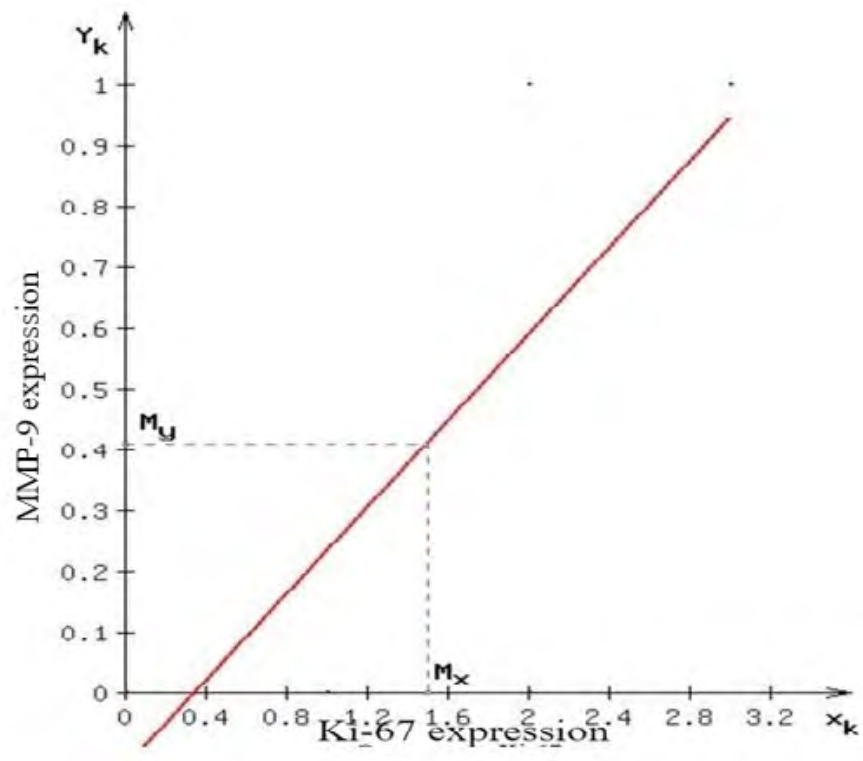

\section{Graph 1. Linear dependence of the expression of the IHC marker MMP-9 and Ki-67}

Expression of apoptosis regulator Bcl-2 also has conflicting values. In some studies, its more pronounced expression in malignant tumors is noted as compared to borderline serous neoplasms of ovaries (Zeren et al., 2014)(Kaygusuz et al, 2011), in others, more pronounced expression is noted in borderline tumors compared to benign and malignant neoplasms (Ozer et al., 2012). The trend towards moderate and pronounced expression of Bcl-2 was observed in the study group $\left(\chi^{2}=3.87, \mathrm{p}<0.05\right)$. In general, the group of SBOT with the subsequent development of LGSC is characterized by an increase in the level of expression of bcl-2, which, in our opinion, may indicate a more aggressive biological behavior of SBOT in this category of patients.

Higher expression of E-cadherin in many studies is observed in borderline tumors compared to serous ovarian cancers. In our study, decrease of E-cadherin expression in the main group compared with the control group was insignificant $(p=0.07)$. The group SBOT without further development of LGSC is characterized by a pronounced and moderate expression of E-cadherin $\left(\chi^{2}=4.36, \mathrm{p}<0.04\right)$, which was observed in $38.46 \%(\mathrm{n}=5 / 13)$ of cases and in $15.38 \%(\mathrm{n}=2 / 13)$ cases, respectively. 
Insignificant associated signs were relationship between tumor diameter and expression of p53 $\left(\mathrm{r}_{\mathrm{s}}=0.149, \mathrm{p}=0.1\right)$ and relationship between tumor FIGO stage and expression of E-cadherin. $\left(r_{s}=-0.284, p=0.1\right)$.

\section{Conclusions}

Currently, issue of diagnosis and prognosis of borderline serous ovarian tumors remains insufficiently studied. The available literature about recommended panel of immunohistochemical markers and their significance as predictors are contradictory. There is also insufficient information about biological behavior of SBOT and their relationship with occurrence of LGSC in future. Clinically, ability to predict appearance of highly differentiated serous ovarian carcinoma in women is very important, because it will allow detecting malignant neoplasms at earlier stages with constant monitoring of patients at risk.

In our study, it was found that the risk of LGSC occurrence within 5 years in women with a history of serous borderline tumors increases with tumor diameter $\geq 10 \mathrm{~cm}$. Also, women who have SBOT proliferative activity $\geq 10 \%$ should be regularly monitored. At risk are also patients with SBOT, detected at FIGO stage II of disease. In women with developed LGSCs, a moderate and pronounced expression of bcl-2 was observed in BOT, which can also serve as a predictor.

Interest in further research into LGSC risk in women with SBOT anamnesis is evident. Evaluation of morphometric parameters in serous borderline ovarian tumors in women with the subsequent development of low-grade serous ovarian carcinoma and correlation of the obtained data with expression of immunohistochemical markers are promising for subsequent studies.

\section{References}

Cirstea A-E., Stepan A.E., Zavoi R.E. \& Simionescu C.E. (2018). Histopathological Patterns of Microinvasion in Ovarian Serous Borderline Tumors. Current Health Sciences Journal, 44(1), 19-22. doi: 10.12865/CHSJ.44.01.03

Corney D.C., Flesken-Nikitin A., Jinhyang C. \& Nikitin A. Yu. (2008). Role of 53 and Rb in ovarian cancer. Advances in Experimental Medicine and Biology, 622, 99-117. doi:10.1007/978-0387-68969-2 9

Davydova I. Yu., CHekalova M. A., Karseladze A. I., Kuznecov V. V., Meshcheryakova L. A., Dedy T. V., CHerkasov E. Yu. \& Zharova A. S. (2021). Seroznye pogranichnye opuholi yaichnikov: sovremennye vozmozhnosti ul'trazvukovoj diagnostiki v monitoringe techeniya bolezni posle organosohranyayushchih operacij [Serous borderline ovarian tumors: modern possibilities of ultrasound diagnostics in monitoring the course of the disease after organ-preserving operations]. Modern onkology, 23 (1), 106-111. Moskow: Konsilium Medikum. [in Russian] doi: $10.26442 / 18151434.2021 .1 .200578$

Gottwald L., Spych M., Kubiak R., Pasz-Walczak G., Piekarski J., Jeziorski A., Sęk P., Szwalski J., Suzin J. \& Tyliński W. (2013). The value of Ki-67 antigen expression in tissue microarray method in prediction prognosis of patients with endometrioid endometrial cancer. Ginekologia Polska, 84(6), 444-449. doi: 10.17772/GP/1602

Grimm F. , Maurus R., Beschorner R., Naros G., Stanojevic M., Gugel I., Giese S., Bier G., Bender B. \& Honegger J. (2019). Ki-67 labeling index and expression of p53 are non-predictive for invasiveness and tumor size in functional and nonfunctional pituitary adenomas. Acta Neurochirurgica, 161(6), 1149-1156. doi: 10.1007/S00701-019-03879-4 
Hauptmann, S., Friedrich K., Redline R., \& Avril S. (2017). Ovarian borderline tumors in the 2014 WHO classification: evolving concepts and diagnostic criteria. In Virchows Archiv, 470 (2), 25-142. doi: 10.1007/s00428-016-2040-8

Herrington, C. S. (Ed.), \& Editorial Board, WHO. C. O. T. (2020). WHO Classification of Tumours Female Genital Tumours. (5th ed.),38-44. Lyon (France): International Agency for Research on Cancer.

Huang H. (2018). Matrix Metalloproteinase-9 (MMP-9) as a Cancer Biomarker and MMP-9 Biosensors: Recent Advances. Sensors (Basel, Switzerland), 18(10), 3249-3268. doi: 10.3390/ S18103249

Jin S. (2005). p53, Autophagy and tumor suppression. Autophagy, 1(3), 171-173. doi: 10.4161/ AUTO.1.3.2051

Kaygusuz E. I., Çetiner H. \& Şahin D. (2011). P53, bcl-2, and nm23 expressions in serous ovarian tumors: correlation with the clinical and histopathological parameters. Turk Patoloji Dergisi, 27(1), 38-45. doi: 10.5146/TJPATH.2010.01045

Li L.T., Jiang G, Chen Q. \& Zheng J. N. (2015). Ki67 is a promising molecular target in the diagnosis of cancer (review). Molecular Medicine Reports, 11(3), 1566-1572. doi: 10.3892/ MMR.2014.2914

Lowe K., Khithani A.S., Liu E.T., Winston T.L., Christian D., Saad J. \& Jeyarajah D. R. (2012). Ki-67 labeling: a more sensitive indicator of malignant phenotype than mitotic count or tumor size? Journal of Surgical Oncology, 106(6), 724-727. doi: 10.1002/JSO.23124

Menon S.S., Guruvayoorappan C., Sakthivel K. M., Rasmi R. R. (2019). Ki-67 protein as a tumour proliferation marker. Clinica Chimica Acta; International Journal of Clinical Chemistry, 491, 39-45. doi: 10.1016/J.CCA.2019.01.011

Ozer H. , Yenicesu G. , Arici S., Cetin M., Tuncer E. \& Cetin A. (2012). Immunohistochemistry with apoptotic-antiapoptotic proteins (p53, p21, bax, bcl-2), c-kit, telomerase, and metallothionein as a diagnostic aid in benign, borderline, and malignant serous and mucinous ovarian tumors. Diagnostic Pathology, 7, 124. doi: 10.1186/1746-1596-7-124

Pârvănescu V., Georgescu M., Georgescu I., Șurlin V., Pătraşcu Ș., Picleanu A. M. \& Georgescu E. (2015). The Role of Matrix Metalloproteinase-9 (MMP-9) as a Prognostic Factor in Epithelial and Lymphatic Neoplasia. Chirurgia, 110(6), 506-510. Retrieved from https:// pubmed.ncbi.nlm.nih.gov/26713823/

Sakata K., Shigemasa K., Nagai N. \& Ohama K. (2000). Expression of matrix metalloproteinases (MMP-2, MMP-9, MT1-MMP) and their inhibitors (TIMP-1, TIMP-2) in common epithelial tumors of the ovary. International journal of oncology, 17(4), 673-81. Retrieved from https://pubmed.ncbi.nlm.nih.gov/10995877/

Sun X. \& Kaufman P. D. (2018). Ki-67: more than a proliferation marker. Chromosoma, 127, 175-186. doi: 10.1007/s00412-018-0659-8

Yu N., Wang N., Liu Y.F., Li Y. Y. \& Zhang T.G. (2017). Expression and clinical significance of Ki-67, E-cadherin, and mesothelin in serous borderline ovarian tumor. European journal of gynaecological pathology, 38(1), 85-90. Retrieved from https://pubmed.ncbi.nlm.nih. gov/29767871/

Zeren T., Inan S., Vatansever H. S. \& Sayhan S. (2014). Significance of apoptosis related proteins on malignant transformation of ovarian tumors: A comparison between Bcl-2/Bax ratio and p53 immunoreactivity. Acta Histochemica, 116(8), 1251-1258. doi: 10.1016/J. ACTHIS.2014.07.007 